\title{
Giant parathyroid adenoma: a rare cause of primary hyperparathyroidism mimicking a carcinoma
}

\author{
Giuseppe Evola $\circledast^{1}$, Martina Tavarelli2, Francesco Roberto Evola $\circledast^{3}$, Giulia Sapuppo $\circledast^{2}$, \\ Gabriella Pellegriti ${ }^{4}$, Romilda Masucci ${ }^{5}$
}

${ }^{1}$ General and Emergency Surgery Department, Garibaldi Hospital, Catania, Italy

${ }^{2}$ Division of Endocrinology, Department of Clinical and Experimental Medicine, Garibaldi-Nesima Medical Centre,

University of Catania, Catania, Italy

${ }^{3}$ Department of Orthopaedics and Traumatology, Cannizzaro Hospital, Catania, Italy

${ }^{4}$ Endocrinology, Garibaldi-Nesima Medical Centre, Catania, Italy

${ }^{5}$ Surgical Oncology, Garibaldi-Nesima Hospital, Catania, Italy

Key words: giant adenoma; parathyroid; primary hyperparathyroidism; hypercalcaemia; pathological bone fracture

Primary hyperparathyroidism (PHPT) is a common disorder due to parathyroid adenoma (PA) in over $85 \%$ of cases. Usually the PA has a weight lower than 3.5 g; rare cases exceeding this weight are defined giant PA. In the literature about 30 cases have been described, and in only three was the parathyroid weight more than 50 grams [1,2]. It is still debated whether giant PA represents a clinical entity different from classical PA. We present a case of a giant PA diagnosed after orthopaedic treatment of a pathological bilateral femur fracture.

A 52-year-old female, complaining of persistent musculoskeletal pains, constipation, and polyuria for two years, underwent an urgent orthopaedic surgical treatment for a spontaneous pathological bilateral femur fracture. At that time blood tests showed mild hypercalcaemia (12.5 mg/dL, normal range 8.4-10.2 mg/dL), extremely high levels of serum parathyroid hormone (2747 pg/dL, normal range 19-65 pg/mL), normal levels of serum phosphorus $(2.8 \mathrm{mg} / \mathrm{dL}$, normal range 2.3-4.7 mg/dL), and hypercalciuria with normal thyroid and kidney function. Neck ultrasound showed a hypoechoic nodule partially extended to the mediastinum, under the left thyroid gland lobe, with smooth borders and homogeneous echogenicity, measuring $4.3 \times 2.8 \times 4.1 \mathrm{~cm}$. Computed tomography (CT) scan of the neck confirmed the presence of a parathyroid gland nodule in the left para-tracheal region with extension to the superior mediastinum (Fig. 1), and left kidney lithiasis. The patient was therefore transferred to an Endocrinology Unit. Medical treatment with saline hydration and bisphosphonates was started, and a few days later, after reduction of calcium serum levels, she underwent parathyroidectomy. During surgical removal of the mass (Fig. 2), intraoperative parathyroid hormone (PTH) measurement showed an important decrease with reduction from $2747 \mathrm{pg} / \mathrm{mL}$ to $428 \mathrm{pg} / \mathrm{mL}$ at 5 minutes and to $303 \mathrm{pg} / \mathrm{mL}$ at 10 minutes. Prophylactic intravenous administration of calcium was started to prevent hungry bone syndrome. Subsequent blood tests showed a progressive reduction of hypercalcaemia and normal parathyroid hormone levels $(20 \mathrm{pg} / \mathrm{mL})$. The patient was discharged on fifth postoperative day in good clinical condition, with normal parathyroid hormone levels and mild hypocalcaemia $(8.0 \mathrm{mg} / \mathrm{dL})$ requiring calcium and vitamin $\mathrm{D}$ supplementation. At pathological examination the giant parathyroid measured $6.5 \times 5.0 \times 3.0 \mathrm{~cm}$ with a weight of $90 \mathrm{~g}$, and macroscopically it showed characteristics similar to PA (Fig. 3) with cellular proliferation without atypia or fibrous bands at histological examination (Fig. 4). At immunohistochemical analysis the Ki-67 index was 3\% . Six months later the patient has normal calcium, phosphorus, and PTH levels and no clinical complications.

We describe the rare case of a giant adenoma in a patient with mild hypercalcaemia and long-term sequelae of chronic hyperparathyroidism (both renal and bone complications). Primary hyperparathyroidism diagnosis is often delayed due to frequent asymptomatic disease and classical symptoms such kidney stones, gastrointestinal and bone manifestation, muscle weakness, fatigue, and depression appearing after many years. On the other hand, acute signs and symptoms of severe 


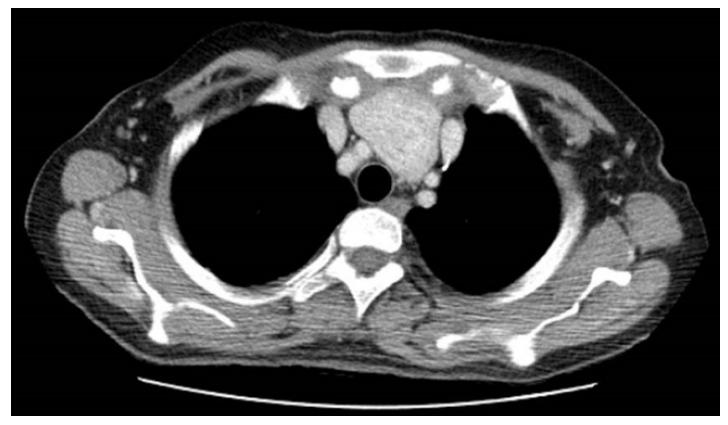

Figure 1. CT scan of the neck. Presence of a parathyroid gland nodule in the left paratracheal region with extension to the superior mediastinum

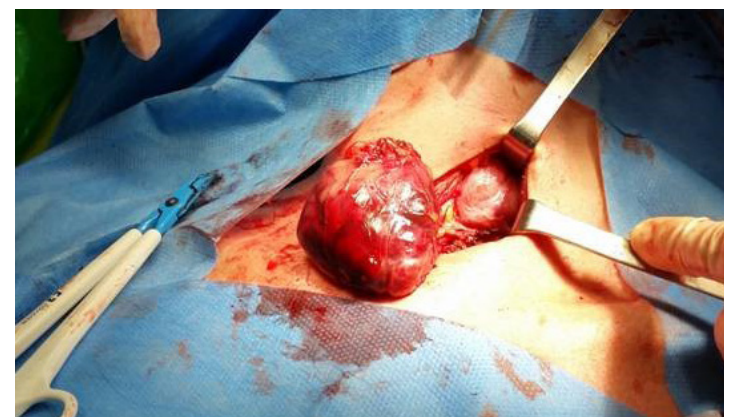

Figure 2. Giant parathyroid adenoma. Left lower parathyroidectomy

hypercalcaemia (polyuria, polydipsia, dehydration, anorexia, nausea, muscle weakness, and changes in sensorium) represent an emergency. Giant parathyroid adenoma is a rare cause of PHPT, which is more frequent in developing countries. In the literature the largest giant adenoma described weighed $145 \mathrm{~g}$ and was found in a Turkish woman with headache and fatigue; another unusual case was described in an Irish 85-year-old woman. High PTH levels require differential diagnosis with parathyroid carcinoma. The histological differentiation between parathyroid adenoma and carcinoma is not simple: various morphological criteria (presence of dense fibrous bands, trabecular architecture, vascular and capsular invasion, mitotic activity, nuclear atypia) have been established to identify a carcinoma; each of them alone has limited sensitivity and specificity, but the simultaneous presence of several of them is common in carcinoma and rare in adenoma. The loss of staining for parafibromin, a protein coded by the HRPT2 gene, and increased values of Ki-67\% are two more elements suggesting a parathyroid carcinoma [3, 4]. It is not clear if giant parathyroid adenomas represent a distinct clinical entity from non-giant adenoma. It should be underlined that there is no correlation between tumour size and severity of hypercalcaemia even in the presence of high PTH levels, as in our patient.

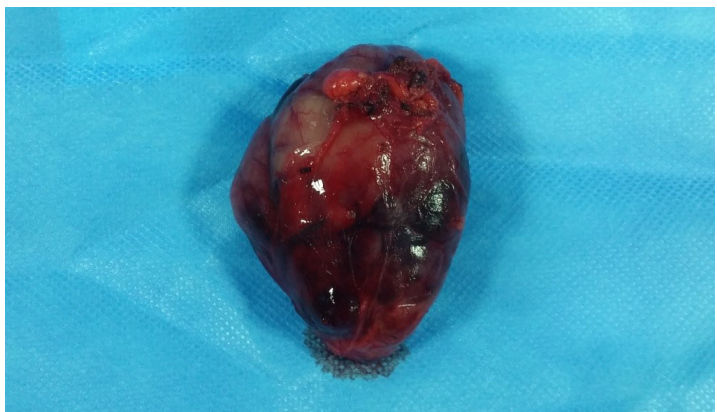

Figure 3. Giant parathyroid adenoma measuring $6.5 \times 5.0$ $\times 3.0 \mathrm{~cm}$ with a weight of $90 \mathrm{~g}$

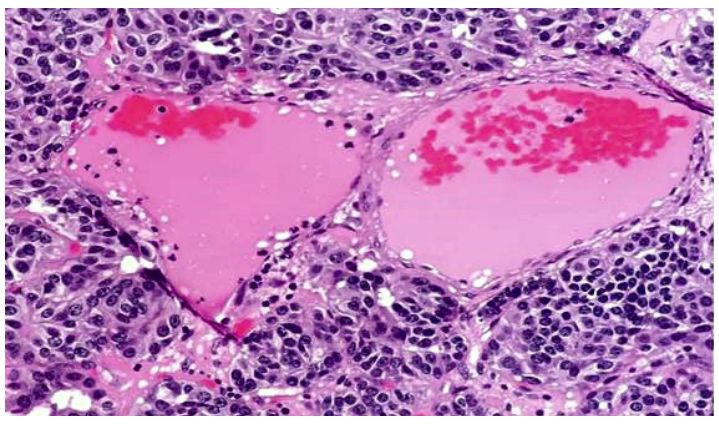

Figure 4. Photomicrograph section of parathyroid adenoma (haematoxylin and eosin, original magnification $\times 20$ )

Moreover, cases of non-functioning giant adenoma probably due to cystic or haemorrhagic degeneration have also been described. In conclusion, PHPT is a rare disease requiring a multidisciplinary approach to avoid acute and chronic sequelae of hypercalcaemia.

\section{Authors' contributions}

G.E. and M.T. contributed equally to the article.

\section{Conflict of interest}

No financial, personal, or professional competing interests exist.

\section{Funding}

None.

\section{References}

1. Lalanne-Mistrih ML, Ognois-Ausse P, Goudet P, et al. Les lésions géantes des glandes parathyroïdes : caractérisation de 26 glandes pesant plus de 3,5 grammes. Ann Chir . 2002; 127(3): 198-202, doi: 10.1016/s0003-3944(02)00717-4, indexed in Pubmed: 11933634

2. Rutledge S, Harrison M, O'Connell M, et al. Acute presentation of a giant intrathyroidal parathyroid adenoma: a case report. J Med Case Rep. 2016; 10(1), doi: 10.1186/s13256-016-1078-1, indexed in Pubmed: 27756436.

3. Castro MA, López A, Fragueiro L, et al. Giant parathyroid adenoma: differential aspects compared to parathyroid carcinoma. Endocrinol Diabetes Metab Case Rep. 2017; 17-0041, doi: 10.1530/edm-17-0041, indexed in Pubmed: 28491324.

4. Karaarslan S, Yurum F, Kumbaraci B, et al. The Role of Parafibromin, Galectin-3, HBME-1, and Ki-67 in the Differential Diagnosis of Parathyroid Tumors. Oman Med J. 2015; 30(6): 421-427, doi: 10.5001/omj.2015.84, indexed in Pubmed: 26675091. 